\title{
Puesta en Valor del Patrimonio Agrícola en Ciudades Intermedias, caso Chillán
}

\author{
Value of Agricultural Heritage in Intermediate Cities, Chillán case
}

Marcela Soto ${ }^{1}$

Anne Helene Escobar Guegen ${ }^{2}$

Recibido: 15 de abril de 2016

Aceptado: 28 de noviembre de 2016

\section{Resumen}

Frente al actual escenario territorial, donde la ruralidad persiste en todas sus expresiones identitarias, sociales y económicas, el suelo agrícola pierde extensión, en una pugna constante por sobreponerse a la extensión urbana. Las modificaciones estructurales del espacio periurbano en las últimas décadas nos presentan la consolidación de los procesos de crecimiento y transformación urbana desiguales, que bajo el modelo neoliberal imperante y persistente, se hacen cada vez más visibles en las ciudades de rango intermedio. Si bien no tienen la escala metropolitana, adquieren velocidades de crecimiento urbano aún mayores. El presente estudio compara la evolución de indicadores relacionados con el desarrollo urbano del territorio para analizar las dinámicas de crecimiento y la pérdida de suelo agrícola. Si bien las ciudades de rango medio promueven el consumo de suelo urbano, existe una oportunidad de resaltar el valor patrimonial y productivo del suelo agrícola.

Palabras clave: ciudades intermedias, crecimiento extensivo, patrimonio agrícola productivo.

\begin{abstract}
From the current territorial scenario, in which the rural persists in all its identity, social and economic terms, the value of productive agricultural land loses extension, in a constant struggle to overcome the urban sprawl. Structural modifications of the peri-urban areas in recent decades present us with the consolidation of the processes of growth and uneven urban transformation, which under the prevailing neoliberal model and persistent, become increasingly visible in cities of intermediate range. While the metropolitan scale have not acquired even greater speeds urban growth. The study compares developments related to urban development of the area concerned to analyze the dynamics of urban growth and loss of agricultural land indicators. While cities midrange promote the consumption of urban land, there is an opportunity to highlight the wealth and productive value of agricultural land.
\end{abstract}

Keywords: intermediate cities, productive agricultural heritage, urban sprawl.

\footnotetext{
${ }^{1}$ Universidad Técnica Federico Santa María. Contacto: marcela.soto@usm.cl

${ }^{2}$ Universidad Técnica Federico Santa María. Contacto: anne.escobarg@gmail.com
} 


\section{Introducción}

En las últimas décadas la transformación y el desarrollo urbanos asociados a procesos globales de reestructuración económica, política y social se han centrado en el estudio de grandes aglomeraciones urbanas por sobre otras áreas de menor tamaño. Sin embargo, hoy en día la atención se ha vuelto a estas últimas, desatando una preocupación cada vez mayor de cientistas sociales, arquitectos y geógrafos por estudiar dichos procesos y su impacto sobre el territorio de estos nuevos espacios de interés: las ciudades intermedias.

Si bien no existe una definición unívoca de dicho concepto, una primera clasificación apunta al volumen de población que varía en diversos intervalos de acuerdo con la nación o territorio. En el caso de Chile, el Ministerio de Vivienda sitúa a las ciudades intermedias en un rango que va de 100.000 a 250.000 habitantes. Sin embargo, el informe acerca de este tipo de ciudades realizado por la Unesco el año 1999 en Lleida, España, señala que la ciudad media o intermedia no puede definirse solo por el tamaño de la población, sino que es más importante el papel y la función que juega la ciudad en su territorio y la influencia y relación que ejerce y mantiene en este.

De esta manera, las ciudades intermedias funcionan como urbes que crean puentes de conexión importantes entre zonas rurales y urbanas que, al no poseer los problemas medioambientales que presentan las megaciudades, se convierten en un claro potencial social y económico. El tamaño y la dimensión urbana a esta escala permitiría que la planificación urbanística pueda ser más eficiente que en una gran ciudad (Llop y Bellet, 2002).

En Chile, al igual que la tendencia mundial, se ha evidenciado un rápido crecimiento de las ciudades intermedias, tanto espacial como demográficamente. Incluso, en las últimas décadas, algunas han crecido a mayores tasas que las grandes ciudades metropolitanas (Sabatini, Cáceres y Cerda, 2001). Si bien la dinámica de acumulación del capital inserto en el territorio a través de infraestructuras físicas es distinta, todas ellas se hacen visibles mediante procesos de producción y consumo del espacio urbano, incidiendo ampliamente en el aumento en las tendencias de exclusión y segregación social como espacial, especialmente para los usos y/o actividades residenciales e industriales (Azócar, Sanhueza y Henríquez, 2003; Bolay \& Rabinovich, 2004).

El capital financiero se materializa en el accionar de los propietarios de la tierra urbana y de los grandes promotores inmobiliarios, mediante lógicas de mercado que diferencian áreas articuladas, valorizadas y dinámicas, por sobre otras áreas marginadas, desestructuradas y debilitadas (Tacoli, 1998). 
Si bien las ciudades intermedias han sufrido transformaciones similares a las metrópolis, el resultado o la expresión territorial no son semejantes. En este sentido, algunas investigaciones destacan que, fundamentalmente, esta diferencia se debe a las características topográficas de las áreas en las cuales se encuentran emplazados los centros urbanos (Borsdorf, Sánchez y Marchant, 2008). Su despegue económico está fuertemente relacionado con la localización, en su ámbito territorial, de inversiones orientadas al mercado internacional, principalmente agrícolas, forestales, pesca, cultivos acuícolas y turismo especializado. No obstante, las evidencias indican que algunas ciudades intermedias, que basan su crecimiento económico en ciertos sectores económicos, han experimentado una notable pérdida de sus recursos naturales de sustento y una degradación de sus condiciones ambientales y sociales (Azócar et al., 2003).

En estas ciudades es donde se hace más evidente la implantación de una lógica de mercado que exige mecanismos y legislaciones que favorecen las iniciativas privadas por sobre las públicas, acentuando así los planes de revitalización urbana en la formación del valor de suelo urbano, con proyectos de infraestructura fuertemente enmarcados por el consumo del suelo agrícola.

Este es el caso de Chillán, capital de la Provincia de Ñuble, que se presenta como núcleo administrativo y prestador de servicios a nivel provincial, ejerciendo una fuerte atracción sobre asentamientos urbanos y su hinterland rural. Dada su localización en el Valle Central de Chile, favorables condiciones climáticas y gran actividad agropecuaria, se ha desarrollado en un entorno rural agropecuario de significativo valor productivo. Esta situación ha presentado grandes desafíos para los planificadores urbanos, quienes deben compatibilizar la extensión del suelo urbano con la aptitud agrícola de los suelos que rodean a la ciudad, además de tender a manejar los desequilibrios territoriales que genera el crecimiento de suelo urbano bajo un modelo fuertemente neoliberal.

Desde esta perspectiva, la presente investigación se centra en el proceso de expansión urbana que ha experimentado Chillán desde 1940 a la actualidad, el cual se ha caracterizado por una fuerte tendencia al crecimiento horizontal y de dispersión y que hoy en día se ve afectado por una serie de problemáticas que imposibilitan su desarrollo de una manera sustentable.

Entendiendo este crecimiento urbano como una expansión que se ha incrementado desmedidamente a expensas de lo rural, se ha realizado una revisión a las modificaciones estructurales del espacio periurbano, el cual ha sufrido una fuerte depreciación y progresiva pérdida de los suelos agrícolas. Como objetivo principal, el artículo busca revalorizar la ruralidad de esta ciudad al identificar un legado patrimonial agrícola del suelo agrícola 
productivo y, con ello, su identidad cultural, desarrollo productivo, equilibrio y sustentabilidad territorial.

\section{Planteamiento del problema}

Uno de los hitos más recordados de Chillán fue sin duda el terremoto que azotó la ciudad en 1939 , donde pereció alrededor del $76 \%$ de la población y un $60 \%$ de las viviendas y edificios fueron derrumbados o quedaron semidestruidos. Adicionalmente, centenares de personas quedaron heridas y la zona debió lamentar grandes pérdidas en la agricultura y la ganadería, debido al daño de las viviendas de campesinos y de las instalaciones agrícolas, silos y bodegas (Urrutia y Lanza, 1993). Este evento se ubica como uno de los desastres naturales más devastadores de la historia de Chillán, que trajo como consecuencia un fuerte impacto en el decrecimiento poblacional de la ciudad, ya que además de causar un alto número de muertes directas, se generaron emigraciones hacia otras ciudades a causa del temor a un nuevo desastre natural de envergadura similar.

Debido a lo anterior, la necesidad de planificar el crecimiento de la ciudad, así como de devolver e impulsar su desarrollo de carácter agrícola condujo, en el año 1952, a la creación del Plan Chillán, reconocido como el primer plan regional integral de desarrollo agrícola en el país.

Luego del terremoto, se dictó la Ordenanza Local de Urbanización que establecía límites urbanos, zonificación de la superficie y altura máxima de los edificios (Miranda, 2010). Desde entonces la ciudad comienza a experimentar una primera etapa expansiva, gatillada por las migraciones campo-ciudad propias de la época, la que genera un crecimiento de carácter horizontal exacerbado en la década de los 80 con el cambio paradigmático a la doctrina neoliberal, la apertura al mercado mundial y, en consecuencia, la integración al proceso de globalización.

Si bien las transformaciones vinculadas con las políticas neoliberales se tradujeron mayoritariamente en una desregularización económica y reestructuración del Estado, las consecuencias en el espacio urbano no tienen precedentes. Un ejemplo de ello es el principio de la liberalización del mercado del suelo bajo el amparo de la Política Nacional de Desarrollo Urbano de 1979. Dicho principio reconocía el suelo urbano como un recurso no escaso y cuyo valor estaba regido por las fuerzas del mercado (Ducci, 1997; Sabatini, 2000). Este proceso permitió a las inmobiliarias transar en el libre mercado los suelos agrícolas, los que fueron incorporados al uso urbano al margen del Plan Regulador, eliminándose los límites al crecimiento urbano al punto de existir predios en la categoría de urbano, pero que todavía mantienen el rol rural (Henríquez, 2006). Lo anterior, sumado a las mejoras de infraestructura urbana y los sistemas de transporte, generó que esta nueva etapa expansiva 
se caracterizase por la dispersión de una ciudad morfológica y socialmente fragmentada, con grandes cifras de pérdida de suelo agrícola que aumentan año tras año.

En el marco de contribuir con el objetivo-país de transformar a Chile en una potencia agroalimentaria, se manejan los mismos principios estratégicos planteados por el Plan de Desarrollo Comunal para acceder a la imagen futura de Chillán: desarrollo productivo, equilibrio y sustentabilidad territorial además de desarrollo económico con equidad social. Sin embargo, el tipo de desarrollo que hoy experimenta la ciudad no se remite a los estándares anhelados. La irreversibilidad de la urbanización y la carencia de una política de protección del suelo agrícola efectiva ha ido en directo desmedro de la sustentabilidad urbana, ya que es justamente la actividad agrícola la que depende de la existencia del recurso suelo y que sostiene la economía de esta ciudad.

Frente a esta problemática (la difícil coexistencia entre los procesos de urbanización y un adecuado desarrollo agrícola), surgen algunas interrogantes pertinentes para lograr la imagen deseada de ciudad: ¿Es sustentable una ciudad que consume su propia base productiva?, ¿cómo o dónde es posible encontrar valor patrimonial agrícola de manera de poder conservarlo?, ¿existen oportunidades territoriales para el desarrollo urbano agrícola?

La investigación que aquí se presenta busca tales respuestas, entendiendo que la evolución de la agricultura en la Región del Biobío, y en particular en la Provincia del Ñuble, está marcada por una situación favorable: se trata de una zona que presenta excelentes condiciones climáticas y edáficas para la producción agrícola y, además, esta actividad da cuenta de la histórica tradición agroproductiva de la zona.

La metodología empleada se basa en la identificación de los procesos de crecimiento y expansión y sus efectos sobre el suelo agrícola. Para ello, se realizó la interpretación de imágenes satelitales de una serie temporal cada 20 años, con análisis taxonométricos en cuanto al consumo del suelo agrícola y detección de otras consecuencias, como su segregación social y espacial la que, al presentar importantes contrastes, permite la identificación de un punto de inflexión ante esta situación y con ello, el reconocimiento del carácter urbanístico y patrimonial agrícola propio de la comuna (áreas de interés paisajístico y valor histórico).

\section{Un crecimiento urbano extensivo}

El estudio del crecimiento urbano se obtuvo de la interpretación de imágenes satelitales secuenciales desde 1940 en adelante, con un intervalo de 20 años, en las cuales se consideraron las superficies urbanas ocupadas por las construcciones de sectores residenciales, industriales y de infraestructura, 
mediante datos cuantitativos entregados por la I. Municipalidad de Chillán. Estos datos indican que la ciudad creció horizontalmente en un $287 \%$ entre el año 1940 y el 2014. Entre 1940 y 1960 la ciudad creció en promedio 11 hectáreas por año, mayoritariamente en torno al casco urbano y con un crecimiento en forma de anillos o ribbonurbanization, asociado a nuevas zonas residenciales (Azócar et al., 2003). Entre 1960 y 2000 la ciudad presentó un crecimiento mayor que en décadas anteriores, con tasas superiores a 20 y cerca de 25 hectáreas por año. Esta etapa expansiva se desarrolló a través de artefactos urbanos (De Mattos, 2002), donde las infraestructuras de transporte o vías interurbanas o urbanas son transformadoras de ejes de cambio. Una de ellas es la zona del bypass (Ruta 5 sur), que se convirtió en un motor de cambio en la localización industrial, para los nuevos barrios residenciales y también para los servicios y equipamientos, es decir, para toda la infraestructura necesaria de un modelo de desarrollo por vías de alta accesibilidad (Graham \& Marvin, 2001).

A partir del año 2000 se produce un notorio incremento en la superficie urbana construida, con un consumo promedio, hasta el año 2014, de 55 hectáreas por año. Esta última etapa expansiva se ha caracterizado por un incremento en el número de parches urbanos. A mayores distancias, tanto del casco urbano como de las vías principales, se han identificado patrones de crecimiento tentacular como también los conocidos como salto de rana o leap frog, que corresponden a un desarrollo urbano discontinuo, donde los enclaves urbanos ocupados por distintos segmentos sociales se emplazan en medio de las inmediaciones rurales (Benguini, Czamanski \& Marinov, 2001; Clawson, 1962; 2007).

Por último, desde el modelo de ciudad difusa definido por Rueda (1996), esto es, la ciudad que se difumina en el campo, ocupando áreas cada vez más extensas, separadas funcionalmente y segregadas socialmente, pero unidas a través de una densa red de carreteras y vías segregadas de transporte privado, es posible identificar un patrón de dirección del crecimiento urbano que es reiterado a través de los años y que se intensifica en la actualidad: la ciudad crece hacia el oriente.

Se presume que esta dirección de la expansión continuará, debido a las limitantes físicas que representan la Ruta 5 sur hacia el poniente y el río Chillán hacia el sur, que se suman al límite comunal hacia este último sector con Chillán Viejo, transformándose en una barrera política que hace que cualquier desarrollo en esta dirección pase a la comuna vecina, como plantea Manuel Durán, arquitecto diseñador del Plan Regulador Comunal. 


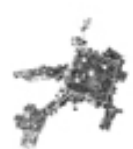

1940 830,99 ha

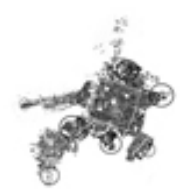

1960

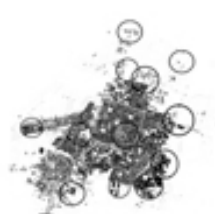

1980

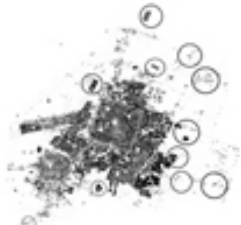

2000 $2.397,01$ ha

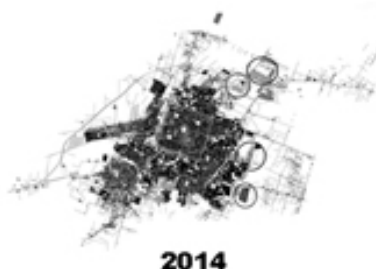

$3.220,23$ ha

Figura 1. Expansión Urbana Chillán.

Fuente: Elaboración propia sobre la base de fotointerpretación de datos proporcionados por la I. Municipalidad de Chillán.

\section{Deterioro del sector agrícola}

Un significativo impacto de estos antecedentes está relacionado con la pérdida de suelos agrícolas de la comuna debido al crecimiento en extensión que ha sufrido la ciudad, un fenómeno irreversible que afecta y continuará afectando la periferia de los centros poblados inmersos en un contexto rural agropecuario de gran valor productivo.

El Informe Ambiental del PRC (Plan Regulador Comunal) afirma que la extensión de suelos urbanos ha sobrepasado el límite urbano comunal vigente, que sumado a la carencia de una normativa urbanística y vialidad estructurante detallada, se ha cuantificado como una disminución notable de la superficie de zonas rurales productivas en las áreas periféricas de la ciudad durante estas últimas décadas.

Para poder cuantificar y espacializar la pérdida de suelo agrícola sufrida en Chillán, se realizó la identificación de los cambios de usos del suelo mediante interpretación de ortofotos pertenecientes a los Estudios Agrológicos de la Región del Biobío de CIREN (Centro de Información de Recursos Naturales, CIREN, 2014).

Los cambios de uso de suelo de aptitud agrícola a zonas residenciales e industriales son los mayores causales del crecimiento urbano fuera de los límites oficiales, tal como se presenta en la Figura 2, donde destaca la cantidad de suelo agrícola perdido por cada zona para los períodos de 1980-2000 y 2000-2014. 


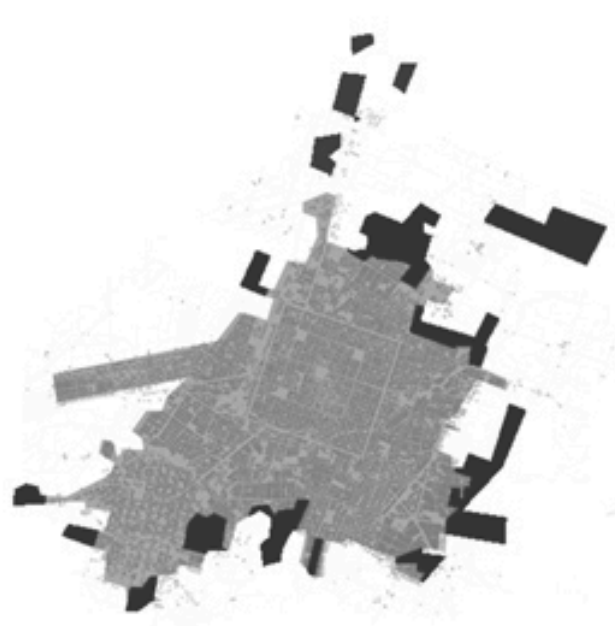

$1980-2000$

$854 \mathrm{Ha}$

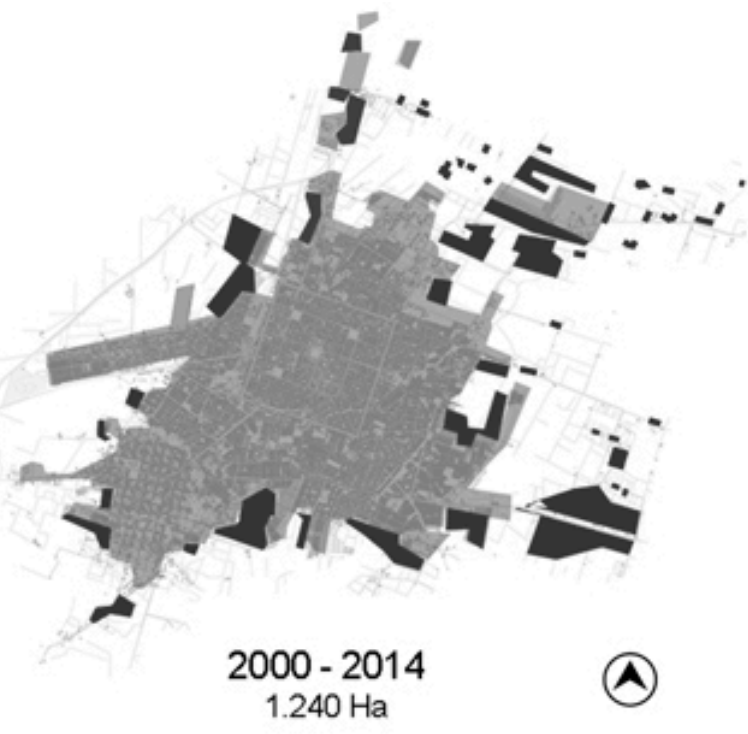

Pérdida superficie agrícola por zona industrial.

Pérdida superficie agricola por zona residencial.

Figura 2. Pérdida superficie agrícola 1980-2000 y 2000-2014.

Fuente: Elaboración propia sobre la base de los Estudios Agrológicos de CIREN, Región del Biobío, 2014.

En términos brutos, la ciudad presentó una pérdida agrícola de 854 hectáreas entre los años 1980 y 2000, cifra que aumenta considerablemente entre los años 2000 y 2014, superando las mil hectáreas, mayoritariamente producto de la expansión urbana de carácter residencial hacia el sector oriente de la ciudad, mientras que en menor medida se presentan pérdidas por cambios de suelo del tipo industrial concentrado en el acceso norte de la ciudad, en torno a la Ruta 5.

Otro factor importante de este fenómeno es el indicador de la calidad del suelo agrícola consumido. Existen ocho categorías de capacidades de uso de suelo, compuesta por tierras adaptadas para cultivo (I, II, III y IV), y tierras de usos limitado (V, VI, VII y VIII) (CIREN, Región del Biobío), las cuales se clasifican en cinco grandes grupos (Tabla 1 ) de manera de lograr medir su consumo según calidad (Figura 3 ). 
Tabla 1

Clasificación del suelo agrícola

\begin{tabular}{|c|c|}
\hline Clases de calidad agrícola & Capacidad de usos de suelo CIREN \\
\hline Muy buena & I y II \\
\hline Buena & III \\
\hline Regular & IV \\
\hline Mala & V y VI \\
\hline Muy mala & VII y VIII \\
\hline
\end{tabular}

Fuente: Elaboración propia.

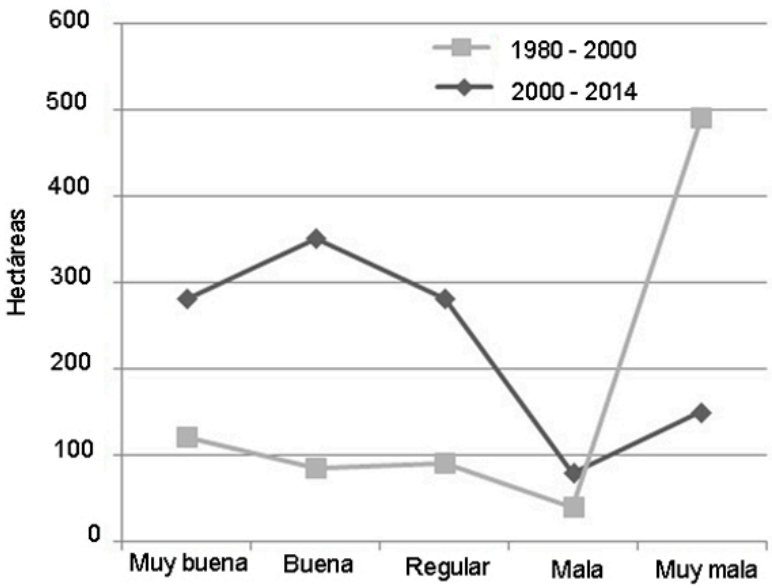

Figura 3. Suelo consumido según calidad agrícola.

Fuente: Elaboración propia sobre la base de los Estudios Agrológicos de CIREN, Región del Biobío, 2014.

En la Figura 3 es posible observar que la superficie consumida durante los últimos años corresponde en mayor medida a suelos de buena calidad agrícola. Esta situación es producto de los nuevos patrones de crecimiento que ha presentado la ciudad, cada vez más distanciados del casco histórico donde se concentran los mejores suelos. En cambio, en los años 1980 y 2000 se consumieron, en mayor medida, suelos de mala calidad agrícola, lo que se atribuye a un crecimiento más compacto, donde los suelos más cercanos a la ciudad corresponden a las clases VII y VIII.

Siendo los suelos de clase I y II muy escasos y, a la vez, el sustento de muchas actividades económicas, su pérdida constituye un daño natural y económico inconmensurable al patrimonio ambiental local (Henríquez, 2013).

Por otra parte, según datos del Banco Central (2007), la dimensión o escala del sector agropecuario regional, medida en términos de Producto Geográfico Bruto (PGB), es decir, el valor (pago a los recursos y factores de producción) de todos los bienes agropecuarios finales generados en la región era de 


\section{URBANISMO}

$\$ 400.000$ millones en el año 2006 , lo que constituía alrededor de un $16,5 \%$ del producto agropecuario nacional: de esta cifra, un $63,3 \%$ prevenía de la Provincia de Ñuble.

Respecto de la evolución del uso del suelo, que genera en alguna medida las variaciones del PGB, en los últimos 15 años los cultivos anuales han experimentado una disminución de superficie sembrada superior a 50.000 hectáreas, aunque continúan siendo el subsector no ganadero de mayor relevancia en ocupación de suelos de aptitud agrícola.

Si bien este deterioro del sector agrícola es también perceptible a nivel regional, ello revierte mayor impacto dentro de la comuna, ya que es justamente la actividad agrícola la que sustenta su economía.

A continuación en la Figura 4 se presenta la distribución de la población de la comuna según ramas de actividad económica ${ }^{3}$, comparando los años 1982 y 2011:

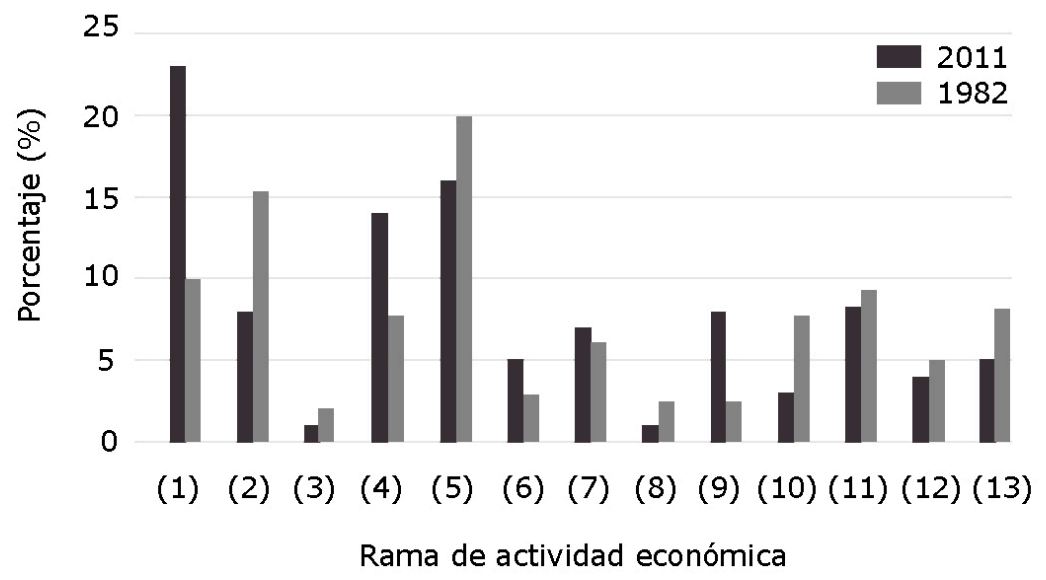

Figura 4. Población según ramas de actividad económica.

Fuente: Elaboración propia sobre la base de datos del Instituto Nacional de Estadísticas de Chile, Censo (1982) y datos del Departamento de Estudios Económicos y Tributarios de la Subdirección de Estudios del Servicio de Impuestos Internos, SII (2011).

3 (1) Agricultura, pesca y minería; (2) Industria; (3) Electricidad, agua potable, gas; (4) Construcción; (5) Comercio; (6) Hotel; (7) Transporte; (8) Financieras; (9) Inmobiliarias; (10) Administración; (11) Enseñanza; (12) Salud; (13) Otros servicios. 
Como se puede apreciar, en el año 1982 la agricultura era superada por el comercio y la industria. Sin embargo, para el año 2011 esta ocupaba a la mayor población económicamente activa de la comuna, la que además iba en un desarrollo creciente. Por lo tanto, esta situación resulta paradójica al encontrarnos con una ciudad que se sustenta en una actividad económica que consume su base productiva, lo que acarrea grandes cifras de pérdida de suelo agrícola año tras año.

\section{Contrastes del sector oriente}

Segregación socio espacial. Durante la década del 70, el Estado se desvincula de las políticas habitacionales, lo que se suma al cambio del modelo económico y a la apertura del mercado de suelos, como lo hemos visto en extensas investigaciones. Ello implicó en la práctica que el Estado cedía su rol principal en la construcción de conjuntos habitacionales a la empresa privada. En Chillán, este fenómeno se evidencia en la construcción de una cantidad importante de viviendas sociales en la periferia oriente y suroriente de la ciudad, como lo son las emblemáticas poblaciones de Los Volcanes y Lomas de Oriente (Espinoza, 2014). Mientras que hacia el norte y nororiente la expansión urbana se realizó con inversiones de gestores inmobiliarios privados, mediante la construcción de conjuntos habitacionales y viviendas unifamiliares de lujo, con amplios jardines y patios, en un entorno rural de privilegio que las convirtió en las denominadas comunidades atractivas (Thomson \& Mitchell, 1996).

Debido a lo anterior se generó un crecimiento urbano significativo hacia la periferia de la ciudad, lo que conllevó finalmente a su fragmentación social, donde la población de menores ingresos se concentró hacia el suroriente, mientras que hacia el nororiente se presentó un crecimiento inmobiliario relevante para grupos socioeconómicos más pudientes.

Esta situación queda graficada en la Figura 5, donde se espacializaron los diferentes grupos socioeconómico predominantes según datos de la distribución de hogares por ingresos entregados por la I. Municipalidad de Chillán, respecto de unidades territoriales establecidas por el estudio de Encuesta Origen Destino (Departamento de Ingeniería y Transporte, Pontificia Universidad Católica de Chile y Secretaría de Planificación de Transporte, SECTRA, 2003). 


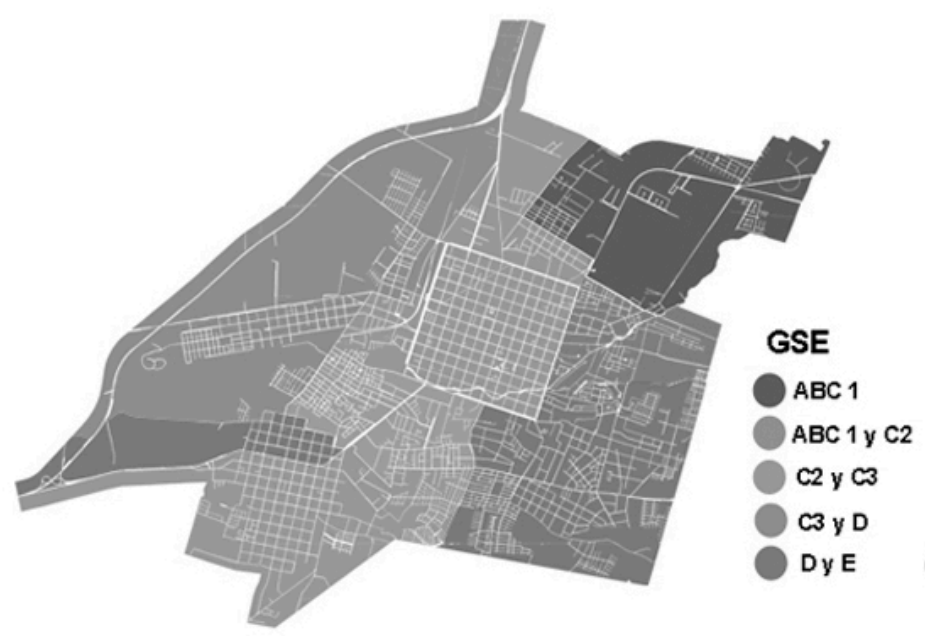

Figura 5. Segregación socioeconómica.

Fuente: Elaboración propia sobre la base de datos de distribución de hogares por ingresos, I. Municipalidad de Chillán y SECTRA, 2003.

Fragmentación del paisaje. La segregación social refleja, a su vez, diferencias visibles en el paisaje. Si bien la población de mayores ingresos se ha asentado en el sector nororiente de la ciudad, lo ha hecho en las modalidades de parcelas de agrado o condominios cerrados, como búsqueda de una mejor calidad de vida y con extensas áreas verdes. Es así que los sectores socioeconómicos alto y medio configuran nuevos barrios residenciales, caracterizados por bajas densidades tanto poblacionales como de infraestructura, presentándose como una ciudad que se construye de fragmentos o partes, muchas veces desconectadas entre sí (Hidalgo, 2010; Inzulza-Contardo, 2016). En contraste, el cambio urbano con altas densidades está asociado a zonas pobres y vulnerables, en este caso, el sector suroriente de la ciudad, donde los intereses políticos del Estado -orientados por una fuerte demanda de viviendas básicas para grupos sociales de bajos ingresosgeneró el desarrollo inmobiliario hacia este sector. Sin embargo, muchas de estas nuevas viviendas no alcanzan los $30 \mathrm{~m}^{2}$ de superficie para grupos familiares integrados, en promedio, por cinco personas, en sectores de la ciudad con carencias de urbanización, equipamiento, infraestructura de transporte y precarias condiciones ambientales (Olave, 1994). 


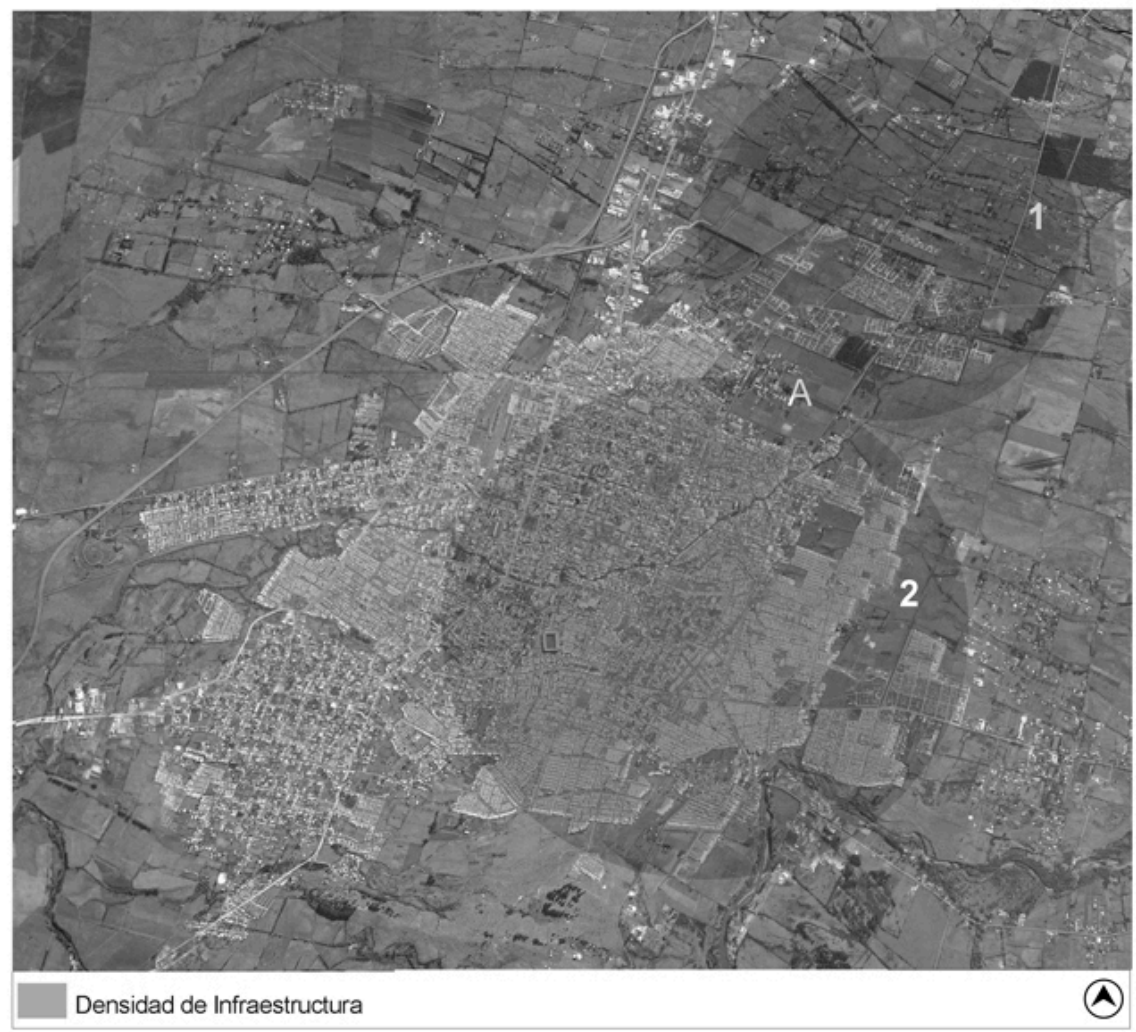

Figura 6. Fragmentación del paisaje.

Fuente: Elaboración propia a partir de fotointerpretación de una imagen de Google Earth.

En la Figura 6 la imagen satelital de la ciudad actual devela el grado de fragmentación física de los nuevos patrones de urbanización sobre el paisaje, donde nuevamente los mayores contrastes se observan hacia el sector oriente. Por su parte, al suroriente se percibe una alta densidad de infraestructura (2) y hacia el nororiente un gran número de parches urbanos se hace presente (1), extendiendo la ciudad mediante fragmentos 0 partes muchas veces desconectados entre sí.

Esta fragmentación, gatillada por el tipo de expansión que ha sufrido la ciudad, también es visible mediante la aparición de islas agrícolas imbuidas en un ambiente urbano, las que probablemente cambien su destino a un uso eriazo o urbano en un futuro cercano, debido a las fuertes presiones inmobiliarias (Henríquez, 2013). Este fenómeno trae consigo un mayor aislamiento entre grupos sociales, lo que favorece la segregación residencial, y a su vez, significa un fuerte impacto ambiental para la ciudad, ya que además de representar grandes pérdidas de superficie agrícola de buena calidad debido a su extensión, la fragmentación espacial y las distancias entre parches promueven, entre otros aspectos, un mayor uso del automóvil, lo que acarrea congestión y contaminación del aire (Anabalón, 2006). 


\section{Punto de inflexión: la oportunidad de revalorizar el suelo agrícola Productivo}

Localización del punto de inflexión. De acuerdo con el estudio previo, la extensión urbana de la ciudad de Chillán ha ido configurando un paisaje altamente fragmentado en su dimensión física y social, lo cual como se ha dicho se percibe de manera acentuada hacia su periferia oriente, la que se ha compuesto básicamente por dos sectores que presentan grandes diferencias tanto físicas como sociales.

Cada uno de estos sectores (sector suroriente y nororiente) ha experimentado un tipo de extensión residencial distinto del otro, lo cual favorece la segregación de la ciudad. Reconocido cada sector como unidades espaciales delimitadas, es posible identificar una brecha entre ambos ${ }^{4}$.

Espacialmente, este punto de inflexión se ubica en la Universidad de Concepción Campus Chillán, la que representa una gran superficie dentro de la ciudad y, además, se caracteriza por poseer extensos paños verdes, propios de un acercamiento del sector, como muestra la Figura 7.

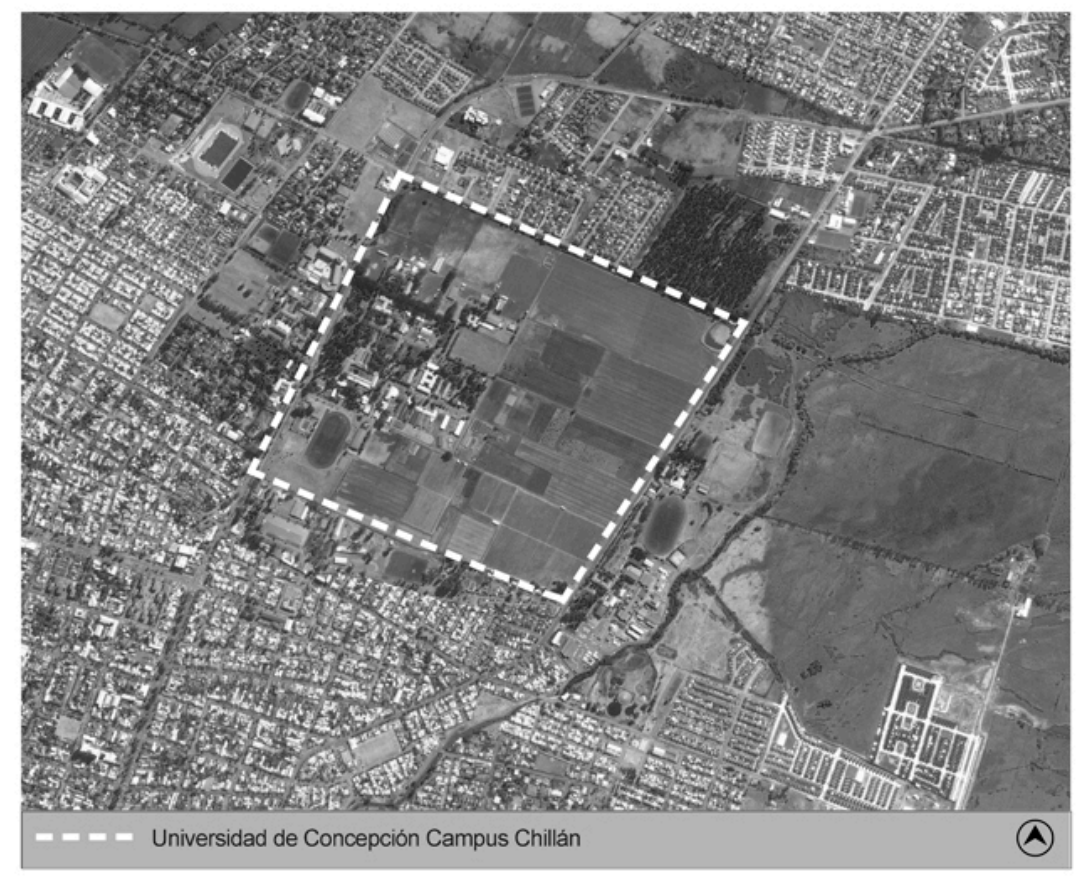

Figura 7. Punto de inflexión.

Fuente: Elaboración propia sobre la base de una imagen de Google Earth.

\footnotetext{
${ }^{4}$ Esto se aprecia en la Figura 6, en la que el punto " $\mathrm{A}$ " es el lugar donde ocurre la transición del paisaje propio entre un contexto y otro.
} 
Historia agrícola local. En el sitio identificado como punto de inflexión de la segregación existe una importante historia local que involucra tanto al desarrollo agrícola en Chillán como a la Universidad de Concepción. En efecto, los terrenos de esta universidad antiguamente correspondían a la Escuela Quinta Agrícola que fuera creada en 1885 como compensación para los hijos de los soldados fallecidos durante la Guerra del Pacífico, y que formó a prácticos agrícolas hasta el año 1952, además de generar un importante dinamismo económico en el sector.

Posteriormente en 1953 se crea, mediante el financiamiento del Ministerio de Agricultura y el Instituto de Asuntos Interamericanos de Estados Unidos, el primer plan regional integral de desarrollo agrícola en el país llamado Plan Chillán, el cual tenía como fin dar a la agricultura el papel que le correspondía cumplir en la economía nacional (Quesada, 2012).

Este programa, que sentó las bases del desarrollo agropecuario local, consistió en la proposición y realización de los siguientes planteamientos:

- Conservación de suelos.

- Extensión.

- Investigaciones.

- Capacitación y maquinaria agrícola.

- Mejoramiento ganadero.

- Salud e higiene rural.

- Planes de divulgación y relaciones públicas (Vergara, 2013).

Uno de los proyectos más relevantes de este programa fue sin duda la creación de la Universidad de Concepción Campus Chillán. Fundada en noviembre de 1954 con la Facultad de Agronomía, además de contar con un prestigioso grupo de profesionales del agro, se sumaron aportes en laboratorios, equipos, biblioteca e instalaciones técnicas, lo que finalmente permitió que en aquellos años este campus asumiera el liderazgo nacional en esta especialidad.

El campus completo de la universidad ocupa una superficie cercana a las 100 hectáreas, organizadas espacialmente mediante una línea imaginaria que divide en forma equitativa la superficie, dejando aproximadamente 50 hectáreas hacia el poniente (donde se concentra la infraestructura del establecimiento y los accesos a la universidad) y otras 50 hectáreas, que corresponden a la "Estación Experimental El Nogal", donde se encuentra una serie de cultivos y una infraestructura menor, tal como lo muestra la Figura 8. 


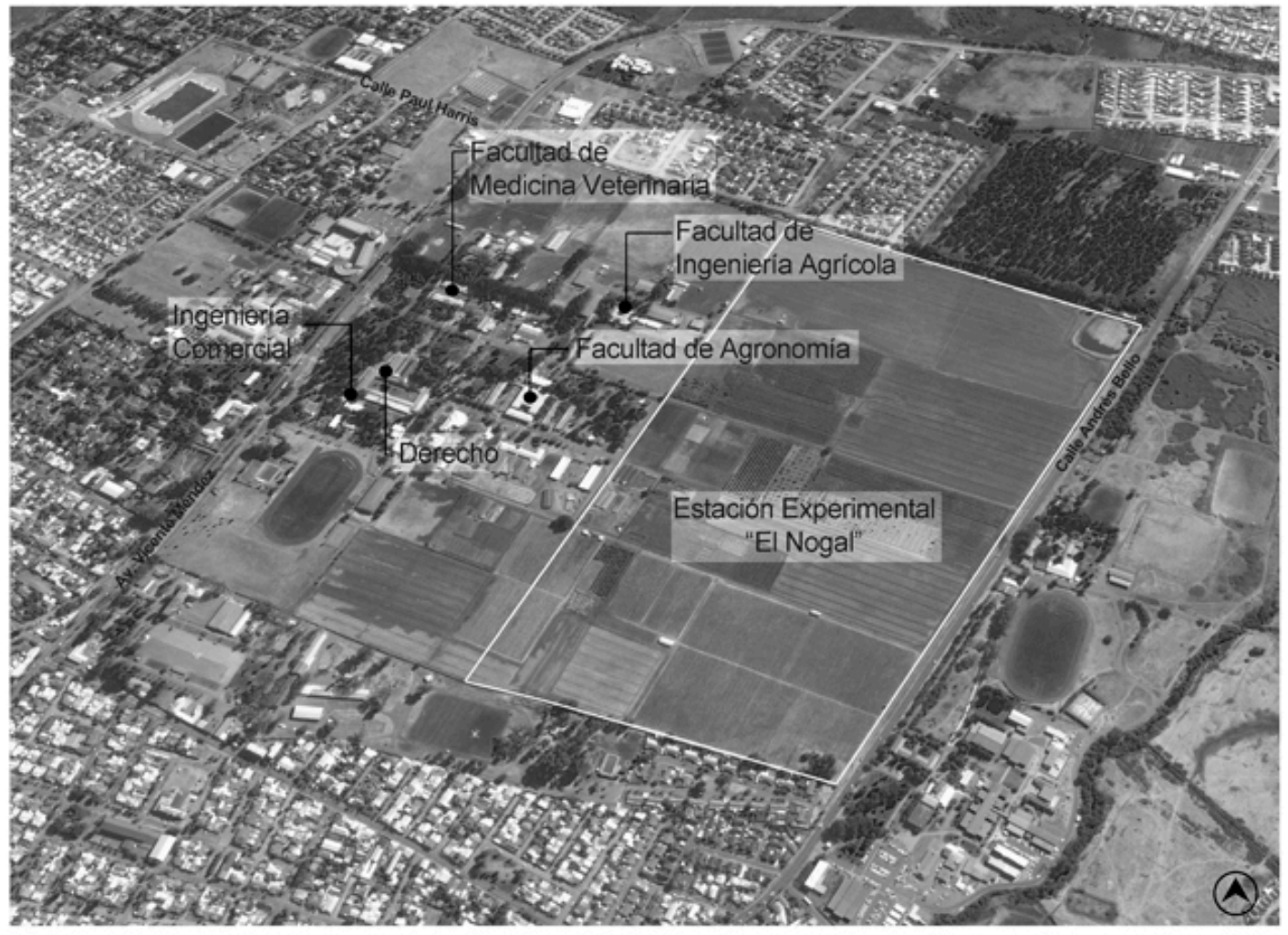

Figura 8. Organización espacial Universidad de Concepción Campus Chillán. Fuente: Elaboración propia sobre la base de una imagen de Google Earth.

La Estación Experimental está compuesta por los cultivos situados en el patio trasero de la universidad y posee fines investigativos y educacionales, lo que la convierte en el único "laboratorio vivo" del país. Los servicios prestados, pertenecientes al Departamento de Producción Vegetal, dentro de la Facultad de Agronomía, se enmarcan en los programas de inocuidad alimentaria y tienen por objetivo proveer información fidedigna a los agricultores $y$ profesionales del agro, que les permita realizar una acertada toma de decisiones respecto de sus cultivos.

\section{¿Amenaza inmobiliaria o conservación dinámica?}

Hoy en día la Estación Experimental está siendo fuertemente amenazada debido a la zonificación de carácter habitacional que le ha otorgado el Plan Regulador Comunal, como muestra la Figura 9. 


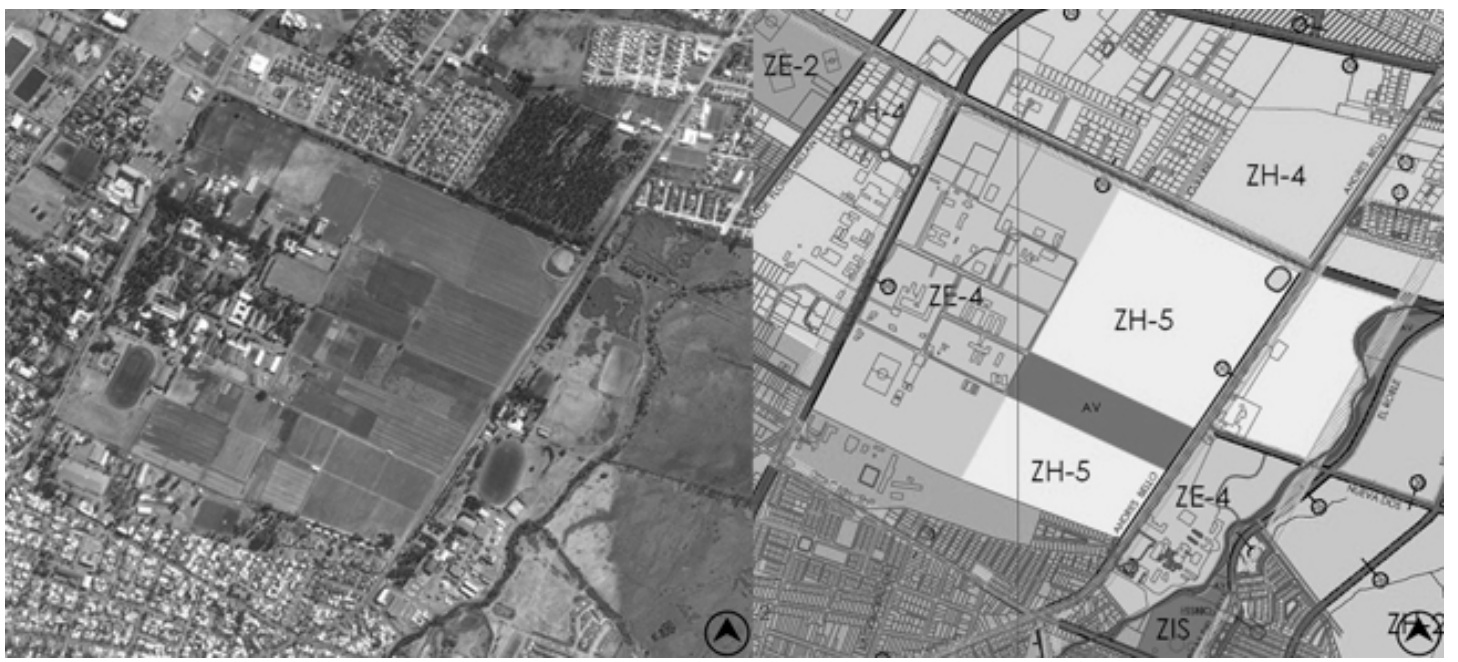

Figura 9. Zonificación Estación Experimental El Nogal.

Fuente: Elaboración propia sobre la base de una imagen de Goole Earth y PRC Chillán.

Esta situación viene a sumarse al crecimiento urbano extensivo de carácter residencial que ha imperado en la zona, hecho que revela la posible sustitución de la estación por nuevos proyectos inmobiliarios, lo cual elimina la posibilidad y el reconocimiento de su valor histórico y patrimonial agrícola.

Es precisamente esta condición la que se presta como principal argumento para debatir en torno a la posible protección de estos suelos, dado el potencial patrimonial agrícola que recae en ellos, puesto que el modelo imperante no es sustentable en el tiempo y la situación amerita usar el factor educativo como bandera.

Finalmente, dado que el funcionamiento de carácter agrícola de estos terrenos es tan importante como los suelos mismos, la proposición de una conservación dinámica se hace necesaria como vía para proteger el sinfín de beneficios que este paño verde aporta a la ciudad, tanto en temas investigativos, educativos y ambientales como también sociales.

\section{Conclusiones}

Hoy en día el tipo de crecimiento urbano que ha desarrollado Chillán repercute fuertemente en la búsqueda de su sustentabilidad y su adecuado desarrollo agrícola. El crecimiento en extensión y dispersión liderado por los usos residenciales ha producido importantes cambios en la composición social de los habitantes que explican, de alguna forma, la vertiginosa transformación física del paisaje urbano. Como resultado de estos procesos se ha constatado una ciudad social y espacialmente fragmentada que, sumado a grandes pérdidas de suelos de buena calidad agrícola, imposibilitan su equilibrio y sustentabilidad territorial. 
El estudio del crecimiento urbano de Chillán reveló una clara dirección del mismo hacia el oriente, por lo que la fragmentación antes mencionada se agudiza en dicho sector, pudiendo percibirse grandes contrastes. Tanto la segmentación socioeconómica como la fragmentación del paisaje revelan notorias diferencias entre los sectores nororiente y suroriente de la ciudad, situación que permitió la localización de un punto de inflexión entre estos: el lugar donde se encuentra ubicada la Universidad de Concepción, bastión y legado patrimonial agrícola de la zona.

En efecto, tanto la Universidad de Concepción Campus Chillán como los terrenos en los que esta se emplaza poseen gran valor histórico agrícola para la ciudad, ya que además de situarse en los terrenos de la antigua Escuela Quinta Agrícola, este campus pertenece a los proyectos realizados por el Plan Chillán que representó la mecanización de la agricultura en la ciudad y permitió el desarrollo de esta actividad tras el devastador terremoto en el año 1939. Sin embargo, las limitaciones del Plan Regulador Comunal que debe compatibilizar la extensión del suelo urbano con la aptitud agrícola de los suelos que rodean a la ciudad, han llevado a parte de estos terrenos de gran valor patrimonial a ser definidos como zonas habitacionales.

Además de representar gran valor histórico, estos suelos zonificados como uso residencial pertenecen a la Estación Experimental El Nogal, donde son ocupados por una serie de cultivos situados como unidades de investigación y desarrollo, lo cual entrega importantes oportunidades de desarrollo, tanto en el ámbito de la docencia e investigación como en la vinculación con el medio. Por lo tanto, una inminente expansión residencial significa una amenaza tanto para el patrimonio de la ciudad como para su potencial desarrollo agrícola.

Ante tal situación resulta imprescindible una intervención para protegerlos, ya que si bien estos terrenos son de gran valor patrimonial agrícola, también lo es la Estación Experimental El Nogal y su funcionamiento, por lo cual cualquier iniciativa no debiera pretender "detenerla en el tiempo", sino más bien potenciar la "conservación dinámica" de la misma, poniendo en equilibrio la conservación y el desarrollo socioeconómico en pos a una economía activa. 


\section{Referencias bibliográficas}

Anabalón, N. (2006). Chillán, una ciudad morfológica y socialmente fragmentada en busca del desarrollo sustentable. Urbano 2006, 9(13), 92-94. Recuperado de http://www.redalyc.org/articulo.oa?id=19813916

Azócar, G., Sanhueza, R., y Henríquez, C. (2003). Cambio en los patrones de crecimiento urbano en una ciudad intermedia: el caso de Chillán en Chile Central. Eure, 29(87), 79-92. https://doi.org/10.4067/s025071612003008700006

Banco Central de Chile. (2007). Estadisticas año 2007. Recuperado de https://is.gd/vcosxc

Benguigui L., Czamanski D., \& Marinov M. (2001). The dynamics of urban morphology: The case of Petah Tikvah. Environment and planning B: Planning and Design, 28(3), 447-460. https://doi.org/10.1068/b2703

Bolay, J.-C. \& Ravinovich, A. (2004). Intermediate cities in Latin America risk and opportunities of coherent urban development. Cities, 21(5), 407-421. https://doi.org/10.1016/j.cities.2004.07.007

Borsdorf, A., Sánchez, R., y Marchant, C. (2008). Aprendiendo de los errores. La necesidad de cambios a la política nacional de vivienda en ciudades intermedias chilenas. Trabajo presentado en el X Coloquio Internacional de Geocrítica, Barcelona.

Centro de Información de Recursos Naturales, CIREN. (2014). Estudio Agrológico Región del Biobío. Descripciones de suelos: materiales y símbolos. Recuperado http://bibliotecadigital.ciren.cl/handle/123456789/25972.

Clawson, M. (1962). Urban sprawl and speculation in suburban land. Land Economics, 38(2), 99-111. https://doi.org/10.2307/3144612

Clawson, M. (2007). Suburban development districts: A proposal of better urban growth. Journal of the American Institute of Planners, 26(2), 6983. https://doi.org/10.1080/01944366008978388

De Mattos, C. (2002). Transformación de las ciudades latinoamericanas: ¿impactos de la globalización? Eure, 28(85), 5-10. https://doi.org/10.4067/s0250-71612002008500001 
Departamento de Ingeniería y Transporte, Pontificia Universidad Católica de Chile y Secretaría de Planificación de Transporte, SECTRA (2003). Encuesta Origen Destino. Santiago: SECTRA.

Ducci, M. (1997). Chile: el lado oscuro de una política de vivienda exitosa. Eure, 69, 99-115.

Espinoza, C. (2014). La dinámica habitacional en Chillán, Chile 1906-2013. Revista INVI, 29(82), 157-187. https://doi.org/10.4067/s071883582014000300006

Graham, S. \& Marvin, S. (2001). Splintering urbanism: Networked infrastructures, technological mobilities, and urban conditions. London: Routledge.

Henríquez, M. (2006). La sustentabilidad socio-ambiental de las ciudades intermedias de Chillán y Chillán Viejo (Tesis para optar al grado de Doctor en Ciencias Ambientales). Universidad de Concepción, Centro de Formación e Investigación en Ciencias Ambientales EULA, Concepción, Chile.

Henríquez, C. (2013). Modelando el crecimiento de ciudades medias. Santiago: Ediciones de la Pontificia Universidad Católica de Chile.

Hidalgo, R. (2010). Los centros históricos y el desarrollo inmobiliario: las contradicciones de un negocio exitoso en Santiago de Chile. Scripta Nova. Revista Electrónica de Geografía y Ciencias Sociales, 331(85). Recuperado de http://www.ub.es/geocrit/sn/sn-331/sn-331-85.htm

I. Municipalidad de Chillan. (2016). Datos comunales. Recuperado de http://www.municipalidadchillan.cl/sitio/

Instituto Nacional de Estadísticas de Chile, INE Censo (1982). Censo de Población Históricos. Recuperado de http://www.ine.cl/canales/usuarios/censos_digitalizados.php

Inzulza-Contardo, J. (2016). Contemporary Latin American gentrification? Young urban professionals (YUPs) discovering historic neighbourhoods. Urban Geography, 37(8), 1195-1214. https://doi.org/10.1080/02723638.2016.1147754

Llop, J. M. y Bellet, C. (2002). Ciudades medias y urbanización mundial. Programa UIA/CIMES. Recuperado de http://www.ceut.udl.cat/wpcontent/uploads/6.BOOK2_.pdf 
Miranda, R. (2010). La frustrada reconstrucción de Chillán por Le Corbusier. La Tercera, 6 de marzo 2010. Recuperado de http://www.latercera.com/noticia/la-frustrada-reconstruccion-de-chillanpor-le-corbusier-2/

Olave, D. (1994). La vivienda, una variable de calidad de vida en la ciudad de Chillán-Chile. Tiempo y Espacio, 4, 95-112.

Quesada, F. (2012). Un modelo para el desarrollo: la cooperación entre Chile y California y el papel del financiamiento público y privado norteamericano. Estudios Avanzados, 18, 11-34. Recuperado de http://www.revistaidea.usach.cl/ojs/index.php/ideas/article/viewFile/1078 $/ 1016$

Rueda, S. (1996). La ciudad compacta y diversa frente a la conurbación difusa. En Ministerio de Obras Públicas, Transporte y Medio Ambiente, MOPTMA (Eds.), Ciudades para un futuro más sostenible. Primer catálogo español de buenas prácticas. Madrid: Comité Español Hábitat II. Recuperado de http://habitat.aq.upm.es/cs/p2/a009.html

Sabatini, F., Cáceres, G. y Cerda, J. (2001). Segregación residencial en las principales ciudades chilenas: tendencias de las tres últimas décadas y posibles cursos de acción. Eure, 27(82), 21-42. https://doi.org/10.4067/s0250-71612001008200002

Sabatini, F. (2000). Reforma de los mercados de suelo en Santiago, Chile: efectos sobre los precios de la tierra y la segregación residencial. Eure, 26(77), 49-80. https://doi.org/10.4067/s0250-71612000007700003

Servicio de Impuestos Internos, SII. (2011). Estudios económicos - tribitarios. Departamento de Estudios Económicos y Tributarios, Subdirección de Estudios. Recuperado de www.sii.cl

Tacoli, C. (1998). Rural-urban interactions: A guide to the literature. Environment and Urbanization, 10(1), 147-166. https://doi.org/10.1177/095624789801000105

Thomson, L. M. \& Mitchel, C. (1996). Counterurbanization in Waterloo Region: The lure of the countryside. En P. Filion, T. Bunting \& K. Curtis (Eds.), The dynamic of the dispersed city: Geographic and planning perspectives on Waterloo Region (303-328). Waterloo: Department of Geography Publications Series 47.

Urrutia, R. y Lanza, C. (1993). Catástrofes en Chile 1541-1992. Santiago: Editorial La Noria. 
REVISTA DE

URBANISMO

ISSN 0717-5051

http://revistaurbanismo.uchile.cl

Revista de Urbanismo N³5 - Diciembre 2016 Departamento de Urbanismo - FAU - Universidad de Chile

Vergara, A. (2013). Plan Chillán and the Transformation of Chilean Agriculture: U.S. Rural Cooperation and Domestic Responses in 1950s Chile. New Orleans: American Historical Association. 